\title{
A clinical study of shoulder arthroscopic rotator cuff repair on the psychological function of patients after operation
}

\author{
Yan Gao ${ }^{1}$, Kailun $\mathrm{Wu}^{2}$, Jiongjiong Guo ${ }^{1}$, Min Cheng ${ }^{1}$ \\ ${ }^{1}$ Department of Orthopedics, the First Affiliated Hospital of Soochow University, Suzhou, China; ${ }^{2}$ Department of Orthopedics, Suzhou Dushuhu \\ Public Hospital (Dushuhu Public Hospital Affiliated to Soochow University), Suzhou, China \\ Contributions: (I) Conception and design: J Guo, M Cheng; (II) Administrative support: M Cheng; (III) Provision of study materials or patients: J \\ Guo; (IV) Collection and assembly of data: Y Gao, K Wu; (V) Data analysis and interpretation: Y Gao, K Wu; (VI) Manuscript writing: All authors; \\ (VII) Final approval of manuscript: All authors. \\ Correspondence to: Min Cheng; Jiongjiong Guo. Department of Orthopedic Surgery, The First Affiliated Hospital of Soochow University, 899 Pinghai \\ Street, Suzhou 215000, China. Email: cmsuzhou@126.com; gjjsuzhou@126.com.
}

\begin{abstract}
Background: Recently, psychological function in patients who underwent rotator cuff repair has been emphasized. However, the relationship of psychological function and clinical outcomes after rotator cuff repair is still poorly understood. This study aims to investigate the impact of arthroscopic rotator cuff repair on psychological status.

Methods: Clinical data were collected retrospectively from 60 patients with rotator cuff tears treated at the First Affiliated Hospital of Soochow University from January 2013 to June 2016. Preoperative and postoperative parameters were calculated and analyzed, including the Visual Analog Scale (VAS) pain score, the University of California, Los Angeles Scale (UCLA), the American Shoulder and Elbow Surgeons scale (ASES), the Simple Shoulder Test (SST), and the Short Form 36 Health Survey (SF-36) scores.

Results: Compared with preoperative scores, the VAS score decreased significantly 1 year postoperatively $(\mathrm{P}<0.05)$. In contrast, the UCLA, ASES and SST scores improved significantly $(\mathrm{P}<0.05)$. The results of the Short Form 36 Physical Component Summary score (SF-36 PCS) and the Short Form 36 Mental Component Summary score (SF-36 MCS) were also improved, which had significant differences before and after arthroscopy surgery $(\mathrm{P}<0.05)$.
\end{abstract}

Conclusions: Arthroscopic rotator cuff repair can significantly improve the psychological function of patients, promote their rapid recovery, and achieve satisfactory results.

Keywords: Rotator cuff tear; shoulder arthroscopy; psychological status

Submitted Dec 25, 2019. Accepted for publication Jul 28, 2020.

doi: 10.21037/apm-19-665

View this article at: http://dx.doi.org/10.21037/apm-19-665

\section{Introduction}

A clinical study of the effect of shoulder arthroscopic rotator cuff repair on the psychological function of patients after operation was performed. Rotator cuff injury is the most common cause of shoulder pain (1). In recent years, with continuous improvements in quality of life and medical care, the incidences of infectious diseases are declining every year. In contrast, the incidence of chronic noncommunicable diseases is rising. Rotator cuff injury, as one of the common chronic diseases, has a serious impact on the quality of life of patients and has caused an immense economic burden to families, communities and even society as a whole (2). Due to a shortage of specific therapies, arthroscopic rotator cuff repair has become the most important procedure to alleviate or cure the disease, especially for patients who are unsuccessful in relieving the symptoms after conservative treatment $(3,4)$. Arthroscopic rotator cuff repair can greatly improve shoulder pain and physiological function. However, most patients with 

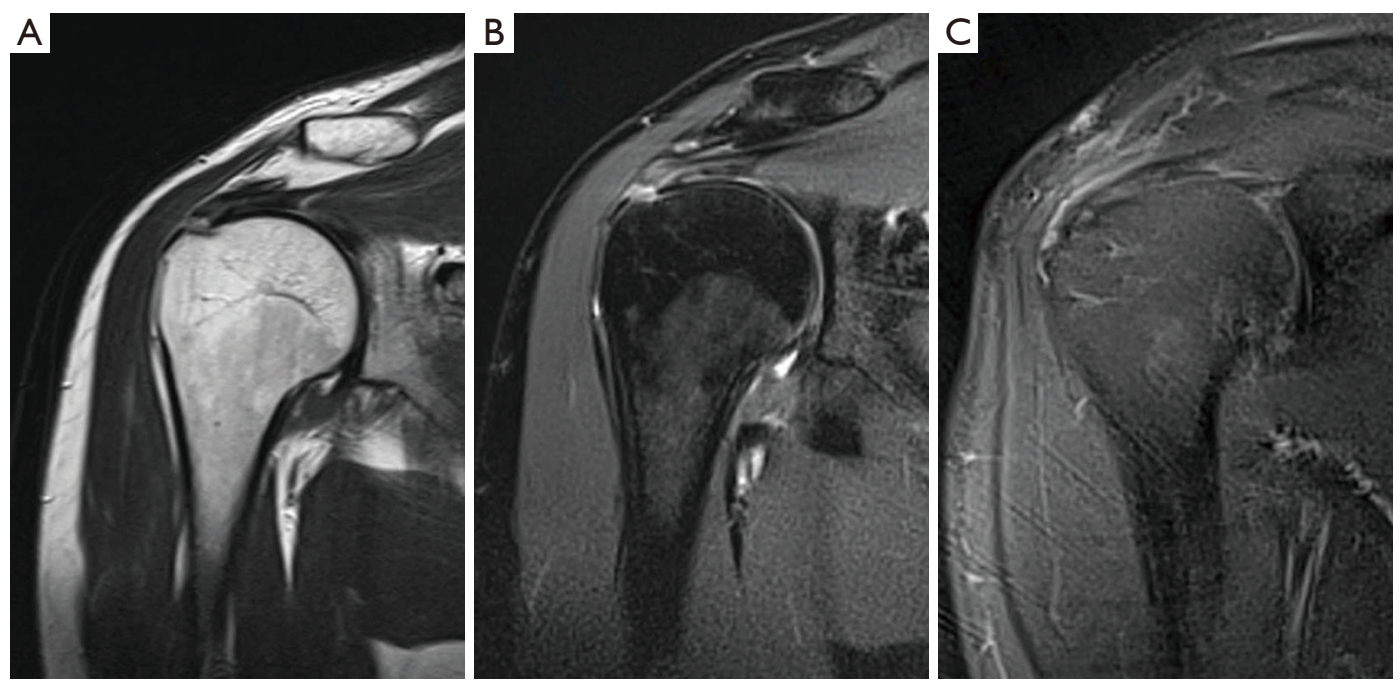

Figure 1 A 35 -year-old male patient suffered from pain in his right shoulder and limited movement after falling down while playing football. (A,B) T2 sequence and fat suppression sequence of MRI suggested that the supraspinatus tendon was torn; (C) the continuity of supraspinatus tendon was found to be intact by MRI at 3 months after operation. MRI, magnetic resonance imaging.

rotator cuff injury will have different degrees of anxiety, insomnia and other psychological disorders before surgery, and the psychological state of patients after surgery is very important for the clinical efficacy of rotator cuff repair $(5,6)$. Therefore, the main purpose of this study was to explore the changes in the psychological function score after rotator cuff repair to evaluate the effect of rotator cuff repair under arthroscopy. We present the following article in accordance with the STROBE reporting checklist (available at http:// dx.doi.org/10.21037/apm-19-665).

\section{Methods}

\section{Patients selection}

From January 2013 to June 2016, 101 patients with rotator cuff injury underwent arthroscopic repair in the Department of Orthopaedics at the First Affiliated Hospital of Soochow University. Of these, 75 patients were eligible for this retrospectively study, and 15 patients were excluded from the study because they were not followed up for 1 year or were unwilling to cooperate with the postoperative evaluation. The study was conducted in accordance with the Declaration of Helsinki (as revised in 2013). The Institutional Review Board for the First Affiliated Hospital of Soochow University approved the protocol for conduct of this study [No. (2020)135]. Informed written consent was obtained from all individual participants about the evaluation of psychological function.

The inclusion criteria for this study were as follows: (I) patients with an magnetic resonance imaging (MRI) clearly diagnosing a rotator cuff tear that was not improved after 3 months of regular conservative treatment, such as medication, injection or physiotherapy (as show in Figure 1); (II) patients with successful repair of the rotator cuff tear by arthroscopic surgery (via single or double row method); and (III) patients with a follow-up time of more than 1 year.

The exclusion criteria of this study were the following: (I) patients with definite contraindications and/or unable to accept a follow-up; (II) patients with partial repair of large rotator cuff tears; and (III) patients with other shoulder diseases.

The average age of the patients was $56.78 \pm 11.64$ years (33-74 years). There were 37 females $(61.67 \%)$ and 23 males $(38.33 \%)$. Among them, 35 cases $(58.33 \%)$ operated on the dominant side of the shoulder, and 25 cases (41.67\%) operated on the nondominant side. Four cases $(6.67 \%)$ were small tears, 15 cases $(25.00 \%)$ were moderate tears, 21 cases $(35.00 \%)$ were large tears and 20 cases $(33.33 \%)$ were massive tears. The average duration of preoperative symptoms was 8.3 months (3-36 months).

\section{Surgical therapy}

All procedures were performed by the same surgeon. The shape, size, type and tendon retraction degree of the rotator 
cuff tear were observed by arthroscopy. According to the size of the rotator cuff tear, 1-2 anchors were implanted. The broken end of the rotator cuff was pulled back to the footprint area and sutured. According to the tear size and bone condition, double-row anchors were added to reinforce the rotator cuff tear. The repair condition of the rotator cuff was observed under arthroscopy when moving the shoulder.

\section{Continuous perioperative management}

Preoperative psychological intervention is defined as follows: responsible physicians and nurses guide patients to emotional talk in the form of inductive questioning and provide preoperative psychological comfort and mental support when necessary;

(II) Preoperative interaction is defined as follows: medical professionals inform patients and their families of the operation information and operation methods, correct their misperceptions and reduce stress;

(III) Postoperative pain intervention is defined as follows: medical professionals should provide each patient with the appropriate analgesic treatment after operation and guide patients to listen to light music and undergo meditation training;

(IV) Rehabilitation training is defined as follows: using the method of sectional training (7), medical professionals should instruct patients to perform resistance internal and external rotation exercises, avoid pain during training, and increase daily exercise (8).

\section{Clinical assessment}

The participants completed the Visual Analog Scale (VAS) pain score, the University of California, Los Angeles Scale (UCLA) score, the American Shoulder and Elbow Surgeons Scale (ASES), the Simple Shoulder Test (SST) score and the Short Form 36 (SF-36) score before surgery and 1, 3, 6 and 12 months after surgery. There are two major categories of the SF-36 scores: the Physical Component Summary (PCS) and the Mental Component Summary (MCS).

\section{Statistical analysis}

The data were analyzed by SPSS, version 17.0. The measurement data were expressed as mean \pm standard deviation (SD). The paired $t$-test and repeated-measures analysis of variance (ANOVA) were used to evaluate the changes in psychological parameters (SF-36 PCS scores) and outcome measurements (VAS score and UCLA, ASES, SST, SF-36 MCS scores). $\mathrm{P}<0.05$ indicated a significant difference between the two groups.

\section{Results}

Sixty patients were followed up for 1 year. The VAS score, UCLA score, ASES score, SST score, SF-36 MCS score and SF-36 PCS score before surgery and 1, 3, 6 and 12 months after operation are shown in Table 1.

By comparing the scores of each group, it can be found that rotator cuff repair can relieve shoulder pain, improve shoulder function and improve the quality of life of patients. The VAS scores decreased significantly at 1, 3, 6 and 12 months after operation, while the UCLA, ASES and SST scores increased gradually. In addition, rotator cuff repair can also improve the psychological state of patients after surgery. The SF-36 MCS and SF-36 PCS scores gradually increased before and 1, 3, 6 and 12 months after operation.

\section{Discussion}

The rotator cuff is an important and dynamic structure for stabilizing the shoulder joint (9). Once a rotator cuff injury occurs, it is easy to cause joint tissue adhesion and shortening, eventually forming scars and affecting shoulder joint function (10). Conventional conservative treatment is often difficult to fundamentally repair and eliminate this inflammatory injury. Although some patients can effectively relieve the symptoms through functional exercise and local blockade treatment, a considerable number of patients show a deteriorating trend, which ultimately leads to the loss of joint function (11).

Arthroscopic rotator cuff repair is often used in our clinical treatment of rotator cuff injury (12). However, due to the unfamiliarity of the operation and the uncertainty of the effect, combined with the long-term pain and lack of functional activity caused by dissatisfaction, patients undoubtedly experience negative emotions that are often manifested as depression, anxiety, insomnia and so on. Relevant studies have shown that patients' expectations and preoperative psychological quality are the key factors affecting postoperative rehabilitation and quality of life $(6,13)$. Therefore, in recent years, the mental health score has become an important indicator for evaluating the 
Table 1 Variations in scores and measurements

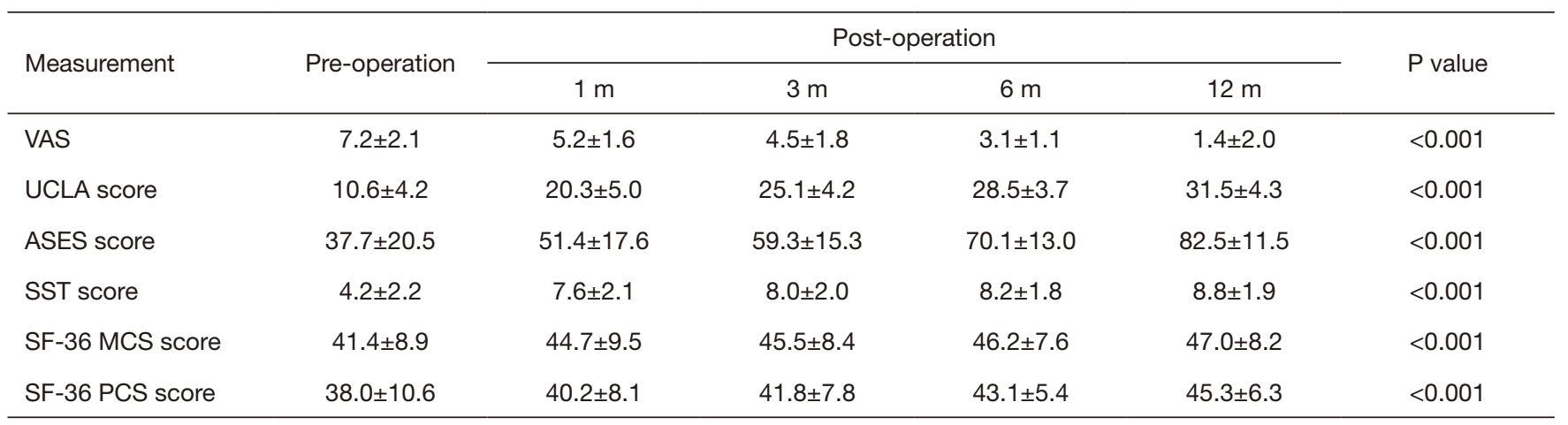

VAS, Visual Analog Scale; UCLA, the University of California, Los Angeles Scale; ASES, the American Shoulder and Elbow Surgeons Scale; SST, Simple Shoulder Test; SF-36, Short Form 36; MCS, Mental Component Summary; PCS, Physical Component Summary.

efficacy of surgery. In this study, we evaluated the effect of arthroscopic rotator cuff repair by assessing the relief of symptoms, functional recovery and psychological status of patients after surgery.

The results of this study show that arthroscopic rotator cuff repair can not only alleviate pain and enhance the activity of affected limbs but also improve the psychological function of patients. In addition, the degree of depression and anxiety experienced by patients decreased significantly 1 month after the operation. However, the results of this study reveal that the psychological state of patients tends to ease from 1 to 12 months after operation, which is not as obvious as that one month after operation. We suspect that this may be related to psychological comfort caused by the surgery (14). In any case, these findings indicate that preoperative depression and anxiety can be effectively alleviated after arthroscopic rotator cuff repair. However, whether preoperative psychological disorders will directly affect the outcome of surgery remains to be further confirmed (15).

This study also has some limitations. First, we did not have a control group, thus the clinical efficacy of nonsurgical treatment could not be compared simultaneously. Second, the follow-up time was relatively short, and the rate of missing follow-up was relatively high (20\%). In addition, our study population had some heterogeneity. The degree of rotator cuff tears ranged from partial tears to giant tears. Finally, due to the complexity of psychological problems, the scale used in this study cannot fully represent the actual situation.

In conclusion, this study found that arthroscopic rotator cuff repair can significantly improve the psychological function of patients, promote their rapid recovery, and achieve satisfactory results.

\section{Acknowledgments}

Funding: None.

\section{Footnote}

Reporting Checklist: The authors have completed the STROBE reporting checklist. Available at http://dx.doi. org/10.21037/apm-19-665

Data Sharing Statement: Available at http://dx.doi. org/10.21037/apm-19-665

Conflicts of Interest: All authors have completed the ICMJE uniform disclosure form (available at http://dx.doi. org/10.21037/apm-19-665). The authors have no conflicts of interest to declare.

Ethical Statement: The authors are accountable for all aspects of the work in ensuring that questions related to the accuracy or integrity of any part of the work are appropriately investigated and resolved. The study was conducted in accordance with the Declaration of Helsinki (as revised in 2013). The Institutional Review Board for the First Affiliated Hospital of Soochow University approved the protocol for conduct of this study [No. (2020)135]. Informed written consent was obtained from all individual participants about the evaluation of psychological function.

Open Access Statement: This is an Open Access article distributed in accordance with the Creative Commons Attribution-NonCommercial-NoDerivs 4.0 International License (CC BY-NC-ND 4.0), which permits the non- 
commercial replication and distribution of the article with the strict proviso that no changes or edits are made and the original work is properly cited (including links to both the formal publication through the relevant DOI and the license). See: https://creativecommons.org/licenses/by-nc-nd/4.0/.

\section{References}

1. Chung SW, Park JS, Kim SH, et al. Quality of life after arthroscopic rotator cuff repair: evaluation using SF-36 and an analysis of affecting clinical factors. Am J Sports Med 2012;40:631-9.

2. Burbank KM, Stevenson JH, Czarnecki GR, et al. Chronic shoulder pain: part I. Evaluation and diagnosis. Am Fam Physician 2008;77:453-60.

3. Mohtadi NG, Hollinshead RM, Sasyniuk TM, et al. A randomized clinical trial comparing open to arthroscopic acromioplasty with mini-open rotator cuff repair for fullthickness rotator cuff tears: disease-specific quality of life outcome at an average 2-year follow-up. Am J Sports Med 2008;36:1043-51.

4. Baysal D, Balyk R, Otto D, et al. Functional outcome and health-related quality of life after surgical repair of fullthickness rotator cuff tear using a mini-open technique. Am J Sports Med 2005;33:1346-55.

5. Cho CH, Seo HJ, Bae KC, et al. The impact of depression and anxiety on self-assessed pain, disability, and quality of life in patients scheduled for rotator cuff repair. J Shoulder Elbow Surg 2013;22:1160-6.

6. Ravindra A, Barlow JD, Jones GL, et al. A prospective evaluation of predictors of pain after arthroscopic rotator cuff repair: psychosocial factors have a stronger association than structural factors. J Shoulder Elbow Surg 2018;27:1824-9.

7. Ross D, Maerz T, Lynch J, et al. Rehabilitation following arthroscopic rotator cuff repair: a review of current literature. J Am Acad Orthop Surg 2014;22:1-9.

8. Monesi R, Benedetti MG, Zati A, et al. The Effects of a Standard Postoperative Rehabilitation Protocol for Arthroscopic Rotator Cuff Repair on Pain, Function, and Health Perception. Joints 2018;6:145-52.

9. Linge VB, Mulder JD. Function of the supraspinatus muscle and its relation to the supraspinatus syndrome. J Bone Joint Surg Br 1963;45:750-4.

10. Murray TF Jr, Lajtai G, Mileski RM, et al. Arthroscopic repair of medium to large full-thickness rotator cuff tears: outcome at 2- to 6-year follow-up. J Shoulder Elbow Surg 2002;11:19-24.

11. Petri M, Ettinger M, Brand S, et al. Non-Operative Management of Rotator Cuff Tears. Open Orthop J 2016;10:349-56.

12. Hultenheim Klintberg I, Karlsson J, Svantesson U. Health-related quality of life, patient satisfaction, and physical activity 8-11 years after arthroscopic subacromial decompression. J Shoulder Elbow Surg 2011;20:598-608.

13. Perruccio AV, Davis AM, Hogg-Johnson S, et al. Importance of self-rated health and mental well-being in predicting health outcomes following total joint replacement surgery for osteoarthritis. Arthritis Care Res (Hoboken) 2011;63:973-81.

14. Potter MQ, Wylie JD, Granger EK, et al. Oneyear Patient-reported Outcomes After Arthroscopic Rotator Cuff Repair Do Not Correlate With Mild to Moderate Psychological Distress. Clin Orthop Relat Res 2015;473:3501-10.

15. Potter MQ, Wylie JD, Greis PE, et al. Psychological distress negatively affects self-assessment of shoulder function in patients with rotator cuff tears. Clin Orthop Relat Res 2014;472:3926-32.
Cite this article as: Gao Y, Wu K, Guo J, Cheng M. A clinical study of shoulder arthroscopic rotator cuff repair on the psychological function of patients after operation. Ann Palliat Med 2020;9(6):4228-4232. doi: 10.21037/apm-19-665 